\title{
Asymptotically null slices in numerical relativity: mathematical analysis and spherical wave equation tests
}

\author{
Gioel Calabrese, Carsten Gundlach, and David Hilditch \\ School of Mathematics, University of Southampton, Southampton, SO17 1BJ, UK
}

(Dated: December 2005, revised April 2006)

\begin{abstract}
We investigate the use of asymptotically null slices combined with stretching or compactification of the radial coordinate for the numerical simulation of asymptotically flat spacetimes. We consider a 1-parameter family of coordinates characterised by the asymptotic relation $r \sim R^{1-n}$ between the physical radius $R$ and coordinate radius $r$, and the asymptotic relation $K \sim R^{n / 2-1}$ for the extrinsic curvature of the slices. These slices are asymptotically null in the sense that their Lorentz factor relative to stationary observers diverges as $\Gamma \sim R^{n / 2}$. While $1<n \leq 2$ slices intersect $\mathscr{I}^{+}$, $0<n \leq 1$ slices end at $i^{0}$. We carry out numerical tests with the spherical wave equation on Minkowski and Schwarzschild spacetime. Simulations using our coordinates with $0<n \leq 2$ achieve higher accuracy at lower computational cost in following outgoing waves to very large radius than using standard $n=0$ slices without compactification. Power-law tails in Schwarzschild are also correctly represented.
\end{abstract}

\section{INTRODUCTION}

Numerical simulations of astrophysical events in general relativity are carried out on a numerical domain that comprises a central strong field zone surrounded by a much larger outer zone which contains no matter, where the fields are weak, and in which gravitational waves are mostly outgoing.

One reason for making the numerical domain large is mathematical. Boundary conditions (BCs) for the continuum initial-boundary value problem which are both compatible with the Einstein constraints and make the problem well-posed have been suggested only recently [1] and are still untested numerically. BCs which are consistent with the constraints (but not proven to be wellposed) 2] have been used successfully in 3D testbed simulations 3 , 4]. Large simulations of astrophysically interesting scenarios have so far been using BCs at finite radius (with the exception of [5], see below) that are incompatible with the constraints, so that finite constraint violations propagate in from the boundary even in the limit of infinite resolution.

A second reason is physical. The central object should be isolated with no incoming gravitational radiation. This cannot be achieved with purely local BCs. For the same reason gravitational radiation cannot be read off reliably close to the source.

The physical problem can be reduced by pushing the outer boundary to larger radius, and the mathematical problem will be alleviated at least by making the domain of dependence of the initial data larger. There is empirical evidence that the dominant error in astrophysics-type GR simulations is from the boundary, and that it can be alleviated for example by using nested boxes mesh refinement to push the outer boundary further out [6, 7, 8$]$.

A radical approach to both the mathematical and the physical problems is to represent a complete asymptotically flat spacetime on the numerical domain. The no ingoing radiation condition can then be imposed exactly at past null infinity $\mathscr{I}^{-}$, and the outgoing radiation can be read off exactly at future null infinity $\mathscr{I}^{+}$. Regularising the field equations at infinity requires embedding the Einstein equations into a much larger system called the conformal field equations [9] , but this system has not yet been used for astrophysical simulations. Alternatively, the Einstein equations can be regularised at $\mathscr{I}^{+}$using retarded time as a null coordinate 10, but this approach cannot be used in the central strong field region because the null cones form caustics.

Finally, space can be compactified on standard spacelike slices going out to $i^{0}$. If the initial data are stationary outside a central region one can then get away with a pseudo-regularisation of the field equations in their discretised form at the compactification boundary 5, 11, 12]. In the continuum equations no gravitational waves reach $i^{0}$ and no evolution takes place there. In the discretised equations spurious radiation must be suppressed by artificial dissipation (or the implicit dissipation of the discretisation) before reaching the compactification boundary.

One key ingredient of the conformal field equations approach is the use of asymptotically null time slices which terminate at $\mathscr{I}^{+}$, together with a compactification of the radial coordinate. It has been suggested to use such coordinates in numerical relativity, but to introduce an artificial outer boundary at some very large radius and to use the standard form of the Einstein equations [13, 14]. Fig. 1 illustrates this approach. Ingoing (backscattered) waves at large radius are increasingly lost in numerical simulations in this approach because they are not resolved on any finite resolution grid. It is sometimes not appreciated that all approaches that put $\mathscr{I}^{+}$at a finite coordinate value, in particular the conformal field equations and the Cauchy-characteristic matching approaches, suffer from the same shortcoming. For example, in Minkowski spacetime with metric $d s^{2}=-d U d V+R^{2} d \Omega^{2}$ the conformal approach would introduce a compactification $U=\tan u, V=\tan v$ on a grid with constant resolution $\Delta u$ and $\Delta v$. This implies $\Delta V=\sec ^{2} \Delta v \simeq V^{-2} \Delta v($ as $V \rightarrow \infty)$, so that 


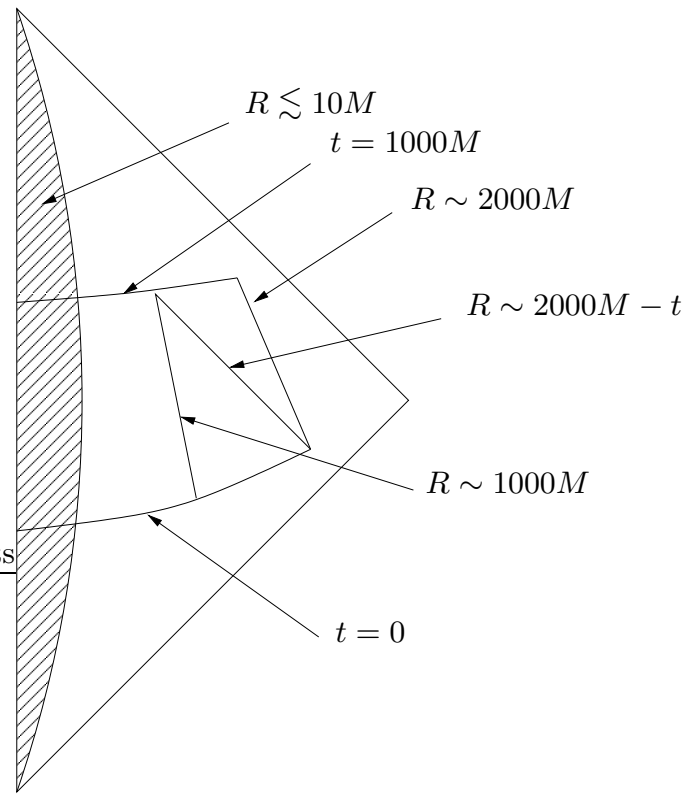

FIG. 1: A schematic spacetime diagram to illustrate that if the artificial outer boundary is pushed out to sufficiently large radius (here $R=2000 M$ ) outgoing gravitational radiation can be read off at very large radius (here $R=1000 M$ ) for a long time (here $t=1000 M$ ) without being contaminated by unphysical BCs at the artificial outer boundary (assuming that no information travels faster than light).

any ingoing waves with period $f$ are no longer resolved for $V \gtrsim(f \Delta v)^{-1 / 2}$. A similar argument holds for the characteristic approach on outgoing null cones.

In this paper, we shall restrict ourselves to the toy model of the wave equation on Minkowski or ReissnerNordström spacetime. In Sect. [I] we investigate in some detail the relationship between radial stretching or compactification, bending up the slices, the coordinate speeds of light, and the global structure of the slices. We propose a one-parameter family of asymptotically null slices comprising flat slices at one extreme and hyperboloidal slices at the other.

In Sect. III we carry out numerical evolutions of an outgoing wave packet in the spherical wave equation in ADM-like form on the Minkowski and Schwarzschild spacetimes. We compare the accuracy and the numerical cost of evolving on different asymptotically null slices with the standard slicing, for realistic values of the numerical parameters. In the Schwarzschild simulations we also look for power-law tails and find that they are correctly resolved. Sect. IV contains our conclusions.

\section{MATHEMATICAL ANALYSIS}

\section{A. Spherically symmetric slices in Minkowski spacetime}

The metric of Minkowski spacetime in standard spherical polar coordinates is

$$
\mathrm{d} s^{2}=-\mathrm{d} T^{2}+\mathrm{d} R^{2}+R^{2} d \Omega^{2},
$$

where $d \Omega^{2} \equiv d \theta^{2}+\sin ^{2} \theta d \varphi^{2}$.

Consider the flat space wave equation as a toy model for the linearised Einstein equations. An outgoing wave $\phi$ emanating from a central region can be approximated as

$$
\phi(T, R, \theta, \varphi) \simeq \sum_{l=0}^{l_{\text {max }}} \sum_{m=-l}^{l} \phi_{l m}(U) R^{-1} Y_{l m}(\theta, \varphi)
$$

for some small finite $l_{\max }$, where the $Y_{l m}$ are spherical harmonics and $U=T-R$ is retarded time. To resolve this, constant spatial resolution $\Delta x, \Delta y, \Delta z$ is not required, but only constant angular resolution $\Delta \theta, \Delta \varphi$. Similarly, constant resolution in $R$ and $T$ separately is not required, but only constant resolution in $U$. If the slices of constant coordinate time $t$ "bend up" such that they approximate slices of constant $U$ asymptotically as $R \rightarrow \infty$, then the radial grid spacing $\Delta R$ can be allowed to diverge in this limit.

To study this quantitatively, we make the change of coordinates

$$
\begin{aligned}
t & =T-F(R), \\
R & =R(r),
\end{aligned}
$$

where $F(R)$ controls the slicing, and $R(r)$ the spatial coordinates on the slices. The new slicing is static, that is, Lie-dragged by the Killing vector $\partial / \partial T$.

The metric in $3+1$ in form in the new coordinates is

$$
\mathrm{d} s^{2}=-\alpha^{2} \mathrm{~d} t^{2}+\gamma_{r r}\left(\mathrm{~d} r+\beta^{r} \mathrm{~d} t\right)^{2}+\gamma_{\theta \theta} \mathrm{d} \Omega^{2},
$$

with the lapse, shift, 3-metric and extrinsic curvature given by

$$
\begin{aligned}
\alpha & =\frac{1}{\left(1-F^{\prime 2}\right)^{\frac{1}{2}}}, \\
\beta^{r} & =-\frac{F^{\prime}}{R^{\prime}\left(1-F^{\prime 2}\right)}, \\
\gamma_{r r} & =R^{\prime 2}\left(1-F^{\prime 2}\right), \\
\gamma_{\theta \theta} & =R^{2} \\
K_{r r} & =-\frac{F^{\prime \prime} R^{\prime 2}}{\left(1-F^{\prime 2}\right)^{\frac{1}{2}}}, \\
K_{\theta \theta} & =-\frac{F^{\prime} R}{\left(1-F^{\prime 2}\right)^{\frac{1}{2}}} .
\end{aligned}
$$


Note that $\alpha \geq 1$. Here and in the following $F^{\prime} \equiv d F / d R$ and $R^{\prime} \equiv d R / d r$. The coordinate light speeds in the radial and tangential directions are

$$
\begin{aligned}
& \left.c_{ \pm} \equiv \frac{\mathrm{d} r}{\mathrm{~d} t}\right|_{\theta, \varphi}= \pm \frac{\alpha}{\sqrt{\gamma_{r r}}}-\beta^{r}= \pm \frac{1}{R^{\prime}\left(1 \mp F^{\prime}\right)} \\
& \left.c_{\theta} \equiv \frac{\mathrm{d} \theta}{\mathrm{d} t}\right|_{r, \varphi}=\sqrt{\frac{-g_{t t}}{\gamma_{\theta \theta}}}=\frac{1}{R} .
\end{aligned}
$$

We can also express the radial coordinate speeds of light $d r / d t=c_{ \pm}$directly in terms of $d R / d T=C_{ \pm}$in the form

$$
c_{ \pm}^{-1}=R^{\prime}\left(C_{ \pm}^{-1}-F^{\prime}\right)
$$

and this relation holds for any spherically symmetric metric.

\section{B. Stability conditions}

We consider a numerical stencil centred at the grid point $x_{0}^{i}$ at time $t_{0}$, and for simplicity of presentation we shift the origin of the coordinates so that $x_{0}^{i}=0$ and $t_{0}=0$. We model the stencil as the square box $-\Delta x^{i} \leq x^{i} \leq \Delta x^{i}$ for $i=1,2,3$. The time step is $\Delta t$, and for simplicity of notation we assume a two-level scheme and a three-point stencil in each spatial direction.

The intersection of the past light cone of the grid point at $x^{i}=0$ and $t=\Delta t$ with the time slice $t=0$ is the ellipsoid

$$
\gamma_{i j}\left(x^{i}+\beta^{i} \Delta t\right)\left(x^{j}+\beta^{j} \Delta t\right)=\alpha^{2} \Delta t^{2} .
$$

Note that the origin of this ellipsoid is shifted from $x^{i}=0$ if $\beta^{i} \neq 0$, and its principal axes are not aligned with the coordinate axes if $\gamma_{i j}$ is not diagonal.

The light ellipsoid fits into the stencil box (the Courant-Friedrichs-Levy (CFL) condition) if and only if in each of the three coordinate directions $i$ the maximal value of $x^{i}$ on the ellipsoid is less than $\Delta x^{i}$ and the minimal value is greater than $-\Delta x^{i}$. The solution of the resulting extremisation problem gives

$$
\frac{\Delta x^{i}}{\Delta t} \geq \alpha \sqrt{\gamma^{i i}}+\left|\beta^{i}\right|
$$

for $i=1,2,3$ (no summation over $i$ ). This result is valid also for non-diagonal $\gamma_{i j}$.

Restricted to spherical symmetry, these conditions become

$$
\begin{aligned}
& \frac{\Delta r}{\Delta t} \geq \frac{\alpha}{\sqrt{\gamma_{r r}}}+\left|\beta^{r}\right|, \\
& \frac{\Delta \theta}{\Delta t} \geq \frac{\alpha}{R} \\
& \frac{\Delta \varphi}{\Delta t} \geq \frac{\alpha}{R|\sin \theta|}
\end{aligned}
$$

The factor $1 / \sin \theta$ in the third condition reflects the standard problem with spherical coordinates on the $z$ axis unrelated to our coordinates and which we ignore here.

The CFL condition, which is necessary for stability, can be written as

$$
\left|c_{+}\right| \leq k \frac{\Delta r}{\Delta t}, \quad\left|c_{-}\right| \leq k \frac{\Delta r}{\Delta t}, \quad c_{T} \leq k \frac{\Delta \theta}{\Delta t}
$$

for $k=1$, where

$$
c_{T} \equiv \frac{\alpha}{R}=\frac{1}{R\left(1-F^{2}\right)^{1 / 2}} .
$$

For typical explicit finite differencing methods for linear hyperbolic equations, a sufficient stability condition is then of the same form for some constant $k$ which depends on the equation and its discretisation (allowing for any number of time levels and size of stencil). This will generalise to any hyperbolic formulation of the Einstein equations. Note that $c_{T} \neq c_{\theta}$ unless $\alpha=1$, and that $c_{T}$ and not $c_{\theta}$ is the relevant quantity for stability.

\section{Slicing, stretching, compactification, and lightcones}

The assumption that the slice is spacelike is equivalent to $F^{\prime}<1$, and the assumption that it becomes asymptotically null is equivalent to $F^{\prime} \rightarrow 1$. For simplicity let us consider functions $1-F^{\prime}$ that decay as a power of $R$ at large $R$, or

$$
1-F^{\prime}(R) \sim R^{-n}
$$

as $R \rightarrow \infty$, where $n>0$. Without loss of generality we assume that the numerical grid is equally spaced in $r$. Then the CFL condition in the radial direction requires that

$$
c_{+} \sim \frac{R^{n}}{R^{\prime}}
$$

is bounded above as $R \rightarrow \infty$. To preserve accuracy, it should also be bounded below. (If $c_{+} \rightarrow 0$ as $R \rightarrow \infty$, an outgoing wave pulse would slow down, become increasingly narrow, and be damped by numerical dissipation.) Therefore we require

$$
c_{+} \sim \frac{R^{n}}{R^{\prime}} \sim \text { const. }
$$

which on integration gives us

$$
r \sim R^{1-n}
$$

This means that for $0<n<1$ we have radial stretching

$$
R(r) \sim r^{1 /(1-n)}, \quad r \rightarrow \infty,
$$

and for $n>1$ we have radial compactification

$$
R(r) \sim(l-r)^{-1 /(n-1)}, \quad r \rightarrow l_{-} .
$$


The special case $n=1$ gives us

$$
R(r) \sim e^{r}, \quad r \rightarrow \infty,
$$

also a case of stretching.

We see that

$$
c_{-} \simeq-\frac{1}{2 R^{\prime}} \rightarrow 0
$$

as $R \rightarrow \infty$ for either stretching or compactification. This means that ingoing wave pulses move very slowly (in $r$ and $t$ ) at large radius, and that they become wider (in $r$ ) as they move in. Although they are insufficiently resolved at large radius, they do not pose a stability problem for the finite differencing scheme. We also see that

$$
c_{T} \sim R^{n / 2-1}
$$

as $R \rightarrow \infty$, and so with constant angular resolution the CFL condition in the tangential directions requires $n \leq 2$.

In summary, the ansatz (22) for the slicing requires $0<n \leq 2$ for the coordinate speeds in all directions to be bounded. Keeping $c_{+}$bounded below as well as above means that $0<n \leq 1$ requires radial stretching and $1<n \leq 2$ requires radial compactification.

As a matter of convention, we always set $F(0)=0$, $R(0)=0$ and $R^{\prime}(0)=1$, so that $c_{+}(0)=1$. For regularity we require $R(r)$ to be odd and $F(R)$ to be even. We introduce a length scale $L$ so that

$$
R^{\prime} \simeq\left(\frac{R}{L}\right)^{n}
$$

as $R \rightarrow \infty$. We demand that $c_{+}(\infty)=1$, which implies

$$
F^{\prime} \simeq 1-\left(\frac{R}{L}\right)^{-n}
$$

Simple closed form expressions for $F(R)$ and $R(r)$ with the required asymptotic behaviour are given in App. A A typical set of coordinate speeds is plotted in Fig. 2]

In summary, compactifying the radius without bending up the slices would mean losing the outgoing radiation. Bending up the slices without radial compactification would increase the radial outgoing characteristic speeds without limit and so violate the CFL condition in the radial direction. Bending up the slices without keeping the angular resolution constant at large radius would violate the CFL condition in the angular directions.

\section{Global structure}

The exponent $n$ can be given a geometric interpretation as follows. There are two preferred families of observers: those normal to the slicing and those along the Killing field $\partial / \partial T=\partial / \partial t$. The speed of the former relative to the latter is

$$
v=-\frac{\beta^{r} \sqrt{\gamma_{r r}}}{\alpha},
$$

and the corresponding Lorentz factor is

$$
\Gamma \equiv \frac{1}{\sqrt{1-v^{2}}} \simeq \frac{1}{\sqrt{2}}\left(\frac{R}{L}\right)^{n / 2}
$$

as $R \rightarrow \infty$.

We see that our $n>0$ slices are asymptotically null in the precise sense that their Lorentz factor diverges as $R \rightarrow \infty$, but surprisingly this does not imply that they reach $\mathscr{I}^{+}$. Consider the null coordinates $V \equiv T+R=$ $t+F(R)+R$ and $U \equiv T-R=t+F(R)-R$ on Minkowski spacetime. Noting that $F(R) \simeq R$ as $R \rightarrow \infty$, we find that $V \simeq 2 R \rightarrow \infty$ as $R \rightarrow \infty$ on a slice of constant $t$. For $U$ on a slice of constant $t$, we find that

$$
\left.\frac{d U}{d R}\right|_{t=\text { const. }}=F^{\prime}(R)-1 \simeq-\left(\frac{R}{L}\right)^{-n}
$$

Integrating this relation over $R$, we find that for $n>1$, $U$ approaches a finite value as $R \rightarrow \infty$ (or equivalently, as we have seen, $V \rightarrow \infty$ ) on a slice of constant $t$, and so the slice reaches a point on $\mathscr{I}^{+}$. For $n \leq 1, U \rightarrow-\infty$ as $V \rightarrow \infty$ on a slice of constant $t$, and so the slice reaches $i^{0}$. If our slices are plotted on the standard conformal diagram, they approach $i^{0}$ horizontally for $n=0$, but tangentially to $\mathscr{I}^{+}$for $0<n \leq 1$. For $1<n \leq 2$ they intersect $\mathscr{I}^{+}$.

For comparison we determine the asymptotic behaviour of the standard conformal compactification of Minkowski [15]. With $U$ and $V$ as above, and $u \equiv$ $(t-r) / 2$ and $v \equiv(t+r) / 2$, this is given by $U=\tan u$ and $V=\tan v$. (For consistency with the rest of this paper $u$ and $v$ have finite range and $U$ and $V$ infinite range). Clearly this is is not of the restricted form we have considered here and which gives a static slicing. But we can show that as $R \rightarrow \infty$ on a slice of constant $t$,

$$
\left.\frac{d T}{d R}\right|_{t=\text { const. }} \simeq 1-\left(\frac{R}{L(t)}\right)^{-2}
$$

which we can interpret as $n=2$ but where $L(t)$ now depends explicitly on the slice. In this compactification $c_{ \pm}= \pm 1$ exactly, and so both ingoing and outgoing waves are represented accurately. The lines of constant $(r, \theta, \varphi)$ are no longer Killing trajectories, and so we cannot implement this gauge choice with "symmetry-seeking" coordinate conditions [16], which include almost all popular coordinate conditions.

\section{E. Reissner-Nordström spacetime}

To obtain asymptotically null slices in ReissnerNordström spacetime, we start from its metric in (for example) Kerr-Schild coordinates $(T, R)$,

$$
\begin{aligned}
d s^{2}= & -(1-f) d T^{2}+2 f d T d R+(1+f) d R^{2} \\
& +R^{2} d \Omega^{2} \quad \text { where } f(R) \equiv \frac{2 m}{R}-\frac{q^{2}}{R^{2}},
\end{aligned}
$$




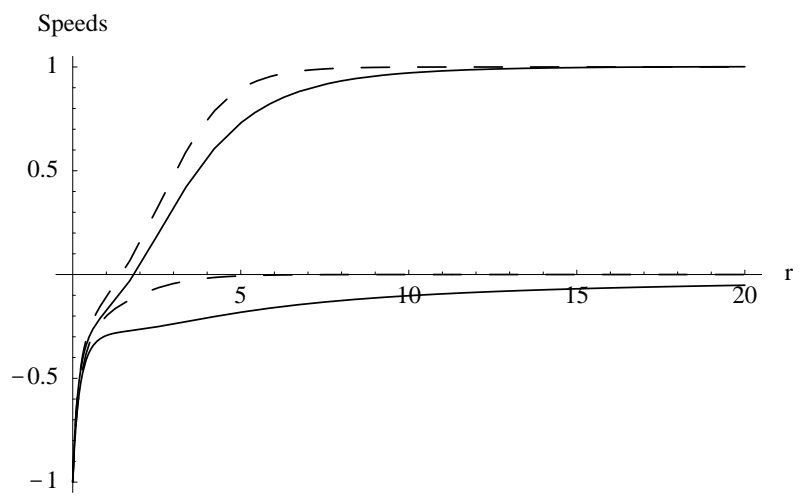

FIG. 2: Coordinate speeds $c_{+}$and $c_{-}$in modified Kerr-Schild coordinates, made asymptotically null with $n=\frac{1}{2}$ (solid lines) and $n=1$ (dashed lines). $L=1$ in both cases.

and make a coordinate transformation of the form (34) to new coordinates $(t, r)$. We choose $R(r)$ to have the asymptotic behaviour (31) with $0<n \leq 2$. From (14) we find that in order to obtain $c_{+}(\infty)=1$, the asymptotic behaviour of $F(R)$ as $R \rightarrow 0$ must now be

$$
F^{\prime}=1+\frac{4 m}{R}+\frac{8 m^{2}-2 q^{2}}{R^{2}}-\left(\frac{R}{L}\right)^{-n}+o\left(R^{-n}\right),
$$

for $0<n \leq 2$. For $n<2$ the $R^{-2}$ term in this expression can be omitted, and for $n<1$ the $R^{-1}$ term can also be omitted, as they give only $o(1)$ contributions to $c_{+}$. Conversely, we see that for $n \geq 1$ the slicing must be corrected for the total mass of the isolated central object. In a dynamic 3D spacetime this mass would presumably be the ADM mass for $n=1$ and the Bondi mass for $n>1$. Although our calculations are limited to spherical symmetry, the analogy between charge and angular momentum suggests that with $n=2$ in $3 \mathrm{D}$ the slicing would have to be corrected for the Bondi angular momentum as well.

Finally we note that (30) holds also in ReissnerNordström spacetime. More precisely,

$$
c_{T} \simeq \frac{1}{\sqrt{2} R}\left(\frac{R}{L}\right)^{n / 2}
$$

for $0<n<2$ with any $m$ and $q$, and

$$
c_{T} \simeq \frac{1}{\sqrt{2} \tilde{L}}, \quad \tilde{L}^{2} \equiv L^{2}+24 m^{2}-4 q^{2}
$$

for $n=2$ as $R \rightarrow \infty$.

\section{F. Characterisation of $n$ in terms of $3+1$ variables}

We have already characterised the slicing geometrically in terms of its Lorentz factor. Another geometric characterisation is in terms of the metric and intrinsic curvature of the slices. Applying the coordinate transformation (34) with the asymptotic behaviour (3138) to the metric (37), we find that for Minkowski with $0<n \leq 2$ and Reissner-Nordström with $0<n<2$,

$$
\begin{aligned}
\alpha & \simeq \frac{1}{\sqrt{2}}\left(\frac{R}{L}\right)^{\frac{n}{2}}, \quad \beta^{r} \simeq-\frac{1}{2}, \\
\gamma_{r r} & \simeq 2\left(\frac{R}{L}\right)^{n}, \quad \gamma_{\theta \theta} \equiv R^{2}, \\
K_{r r} & \simeq-\frac{n}{\sqrt{2} L}\left(\frac{R}{L}\right)^{\frac{3 n}{2}-1}, \\
K_{\theta \theta} & \simeq-\frac{L}{\sqrt{2}}\left(\frac{R}{L}\right)^{\frac{n}{2}+1}, \\
K & \simeq-\frac{n}{2 \sqrt{2} L}\left(\frac{R}{L}\right)^{\frac{n}{2}-1},
\end{aligned}
$$

in the limit $R \rightarrow \infty$. (For $n=2$ with $m, q \neq 0$, the leading order coefficients change from the ones given here). $R$ is defined geometrically as the area radius. To leading order in $R$, there is no effect from the mass $m$. As $R \rightarrow \infty, K=K^{r}{ }_{r}+2 K^{\theta}{ }_{\theta}$ is dominated by $K^{r}{ }_{r}$ for all $n$. For $n=0, K$ vanishes identically, for $0<n<2$ it goes to zero as $R \rightarrow \infty$, while for $n=2$ it approaches a constant.

\section{NUMERICAL EXPERIMENTS}

\section{A. Numerical method}

We solve the wave equation restricted to spherical symmetry on Minkowski and Schwarzschild spacetime. Our initial data is an outgoing Gaussian pulse near the origin (but outside the black hole). On Minkowski spacetime the problem has an overall scale-invariance, which we fix by setting the width of the pulse to $\Delta R \sim 1$. In the Schwarzschild simulations, we also set $m=1$.

In astrophysical simulations we would need to maintain a fairly constant resolution in a finite inner region where the dynamics take place. As a testbed for 3-dimensional simulations, we therefore use a resolution of $\Delta r=0.1$, which, with our convention $R^{\prime}(0)=1$, corresponds to $\Delta R \simeq 0.1$ in the central region, or $\sim 10$ grid points over the width of the pulse. We therefore choose $L$ or $l$ so that $R^{\prime}(r) \simeq 2$ at $R=10$, which means that resolution at that radius is still half of that at the centre.

We solve the wave equation in the continuum form (B3IB4). We use 2nd and 4th-order accurate finite differencing schemes proved to be stable in [17], with a Courant factor of $\Delta t / \Delta r=0.4$. A regular origin is dealt with by imposing regularity, and taking appropriate limits in the right hand sides. For black hole excision we use outflow BCs.

We estimate the error and check that the results converge to the expected order by comparing the three resolutions $\Delta r=0.1,0.05$ and 0.025 . An example is Fig. 3. where we plot the local error $e\left(r, t_{*}\right)$, where 
$t_{*} \equiv t\left(R=R_{*}, T=R_{*}\right)$ is the value of $t$ when the maximum of the Gaussian pulse is at physical radius $R=R_{*}$ in a given slicing.

\section{B. Outer boundary treatment}

If the outer boundary is at $R_{\max }=\infty$, we impose BCs from the exact solution. For the 2nd-order accurate code, this simply means imposing $\phi=\Pi=0$ at the outermost grid point $i=N$ where $R=\infty$. In the 4 th-order accurate code on Minkowski we also impose the exact solution at $i=N-1$. On Schwarzschild, where no exact solution exists, we copy $R \phi$ and $R \Pi$ from $i=N-2$ to $i=N-1$. (This is only first-order accurate, but in practice does not affect results away from the boundary.)

If the outer boundary is at finite physical radius $R_{\max }$, we impose maximally dissipative BCs (MDBCs) on $\phi$ and $\Pi$, with lower-order terms representing the $1 / R$ falloff. This continuum BC perfectly represents outgoing waves in spherical symmetry on flat spacetime, but in curved spacetime and/or for non-spherical waves gives rise to an unavoidable small continuum reflection. The finite differencing implementation of this $\mathrm{BC}$ also gives rise to numerical reflections which converge away with increasing resolution.

With $n>0$ the outer $\mathrm{BC}$ is "almost" an outflow boundary. A second possible $\mathrm{BC}$ is therefore to increase the radial shift by a small amount, leaving the other metric coefficients unchanged, to turn the outer boundary into a genuine outflow boundary. The background spacetime is then no longer a vacuum solution of Einstein's equations but the error is small. This is a simple variant of Misner's idea 14] of introducing a small cosmological constant to create a cosmological horizon that represents a null outer boundary at constant $R$.

As a third BC, we have also tested an outer buffer zone in which $c_{+}$goes smoothly to zero. This can be achieved by reducing $n$ of the slicing and/or increasing $n$ of the radial coordinate transformation. In either case the effect is that outgoing waves become "blue-shifted" with respect to the grid spacing and are then suppressed by artificial dissipation. We consider the buffer not as part of the physical spacetime but rather as a BC. For convenience of implementation, with $n \geq 1$ in the physical region we reduce $n$ for the slicing in the buffer, and with $n<1$ in the physical region we increase $n$ of the radial coordinate transformation (to a value $>1$ ) in the buffer. This means that for all values of $n$ in the physical region the buffer goes to $\infty$.

\section{Spherical wave equation on Minkowski}

We evolve an outgoing scalar field pulse with different slicings with the aim of quantifying the error and establishing what fraction of grid points can be saved by using asymptotically null coordinate slices, and what effect

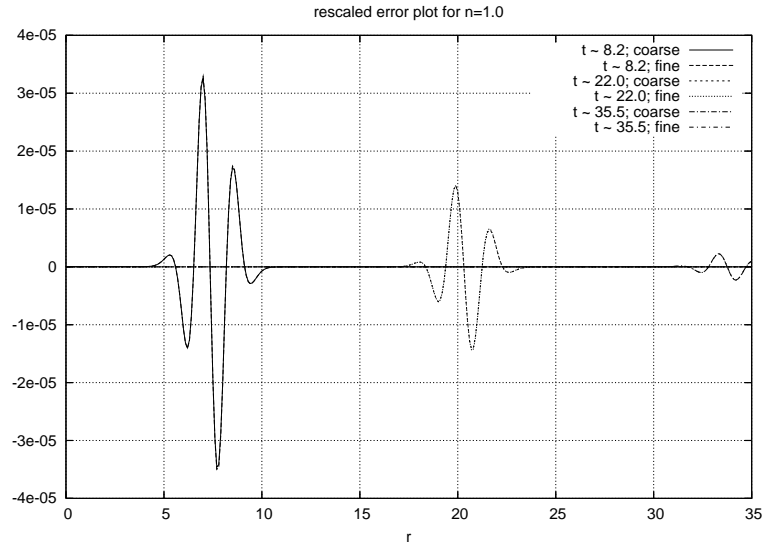

FIG. 3: The Error in $\phi$ with $n=1$ and $L=5.77$, at $R_{*}=10$, 100 and 1000. $e\left(t, r_{*}\right)$ at $\Delta r=0.1$ and $16 e\left(r, t_{*}\right)$ at $\Delta r=0.05$ are both plotted, but are indistinguishable here, indicating 4thorder convergence. The absolute error $e$ decreases with $R_{*}$, but the relative error $e / R$ increases.

they have on the error as the wave goes out to very large $R$. We present results for $n=1 / 2,1,3 / 2,2$. The results for $n \leq 1$ have been obtained with MDBCs at $R \gtrsim 1000$, and the results for $n>1$ by imposing $\phi=\Pi=0$ at $\mathscr{I}^{+}$ and, in 4th-order accuracy, the exact solution at the grid point just inside the boundary.

We evolve up to the time when the outgoing wave reaches the outer boundary. Until then solutions for all $n$ converge with the expected order (2nd or 4 th), and so we have an accurate estimate of the numerical error. The number of grid points required to reach a given physical radius $R_{\max }$ and the norm of the finite differencing error when the wave has gone out to three selected values $R_{*}$ of $R$ are summarised in Table【 Fig. [3 is a typical example of the error plots.

The larger the value of $n$, the smaller the number of grid points required. The error when the wave reaches $R=10$ is similar for all values of $n$ as one would expect from the fact that all grids are similar in the central region $R \lesssim 10$, but by the time the wave reaches $R=1000$ all $n>0$ slicings do better than $n=0$, with $n=1$ an order of magnitude more accurate than $n=0$. We believe that this could be explained by the much smaller number of time steps required for the wave to reach $R=1000$, for example 338 for $n=1$ compared to 10000 for $n=0$. This would tend to reduce phase error, and in dissipative schemes also amplitude error.

\section{Spherical wave equation on Schwarzschild}

Fields in a curved asymptotically flat spacetime generically decay with power law tails because of backscatter [18]. In order to see how well our coordinates can cope with such small physical effects at large physical radii, we evolve the spherical wave equation on the Schwarzschild 


\begin{tabular}{|c|c|c|c|c|c|c|c|c|c|c|}
\hline Slice & \multicolumn{2}{|c|}{ Grid points to cover } & \multicolumn{2}{|c|}{ Time steps to reach } & \multicolumn{3}{|c|}{$\left|e\left(\cdot, t_{*}\right)\right|$ with 2 nd order code at } & \multicolumn{3}{|c|}{$\left|e\left(\cdot, t_{*}\right)\right|$ with 4 th order code at } \\
\hline$n=0$ & 10000 & $\infty$ & 25000 & $\infty$ & $1.8 \cdot 10^{-3}$ & $1.7 \cdot 10^{-3}$ & $5.0 \cdot 10^{-4}$ & $2.0 \cdot 10^{-5}$ & $1.9 \cdot 10^{-5}$ & $1.9 \cdot 10^{-5}$ \\
\hline$n=\frac{1}{2}$ & 893 & $\infty$ & 2300 & $\infty$ & $1.5 \cdot 10^{-3}$ & $7.5 \cdot 10^{-4}$ & $2.5 \cdot 10^{-4}$ & $1.7 \cdot 10^{-5}$ & $1.2 \cdot 10^{-5}$ & $4.5 \cdot 10^{-6}$ \\
\hline$n=1$ & 338 & $\infty$ & 1000 & $\infty$ & $2.3 \cdot 10^{-3}$ & $8.0 \cdot 10^{-4}$ & $1.5 \cdot 10^{-4}$ & $3.2 \cdot 10^{-5}$ & $1.3 \cdot 10^{-5}$ & $2.0 \cdot 10^{-6}$ \\
\hline$n=\frac{3}{2}$ & 207 & 224 & 624 & 667 & $2.5 \cdot 10^{-3}$ & $1.1 \cdot 10^{-3}$ & $2.5 \cdot 10^{-4}$ & $2.3 \cdot 10^{-5}$ & $2.4 \cdot 10^{-5}$ & $4.8 \cdot 10^{-6}$ \\
\hline$n=2$ & 165 & 167 & 390 & 418 & $1.5 \cdot 10^{-3}$ & $6.0 \cdot 10^{-4}$ & $5.0 \cdot 10^{-4}$ & $1.8 \cdot 10^{-5}$ & $1.0 \cdot 10^{-5}$ & $4.0 \cdot 10^{-6}$ \\
\hline
\end{tabular}

TABLE I: Computational costs and errors in evolving a spherical wave on Minkowski spacetime for different slicings. As the outgoing wave has amplitude $1 / R$, a suitable measure of the relative error would be $e / R$.

background, on the horizon-penetrating asymptotically null slices derived above. A priori it is not clear if tails will be represented correctly or not: on the one hand they are caused by backscattered ingoing waves, on the other hand their frequency in advanced time is low.

The numerical algorithm is the same as for our Minkowski experiments, except for the boundaries. The regular origin is replaced by an outflow (excision) boundary inside the black hole. We do not see large errors or any sign of instability associated with the excision boundary. We use the 4th-order accurate scheme with the artificial dissipation parameter $\sigma=0.007$. The results depend only weakly on $\sigma$ (including $\sigma=0$ ).

At the outer boundary we use three types of BC. The first corresponds to what we did in Minkowski spacetime, that is, we impose MDBCs at finite $R_{\max }$ or "exact" $\mathrm{BCs}$ at $\infty$. This gives good tail results if we put the outer boundary far enough out. As a second type of boundary we have increased the shift near the boundary slightly to obtain an outflow BC. As a third type of BC we have added a $20 \%$ buffer in which $c_{+}$goes to zero and $R$ reaches infinity (even if $n \leq 1$ in the physical region). The case $n=0$ with such a buffer is similar to compactification at $i^{0}$ used in [6, 7], [8].

As we do not have an exact solution, we have read off initial data $\phi$ and $\Pi$ on a given initial slice from what would be an exact solution on Minkowski where the Minkowski coordinates $T$ and $R$ are identified with KerrSchild coordinates. This means that we have slightly different initial data for different slicings. We have therefore used a high resolution simulation on each slicing as a reference solution to decide when power-law tails are lost. We see that at $R=500$ the power is not exactly 3 as expected for constant $R$, but varies between 3 and 2 (the power expected at $\mathscr{I}^{+}$).

The errors and computational costs on the domain of dependence of the initial data are similar to those given in Table 【 Therefore we concentrate on power law tails at late times. Our results are summarised in Table III To check for tails, we plot $\phi(t)$ for "observers" at $R=10$

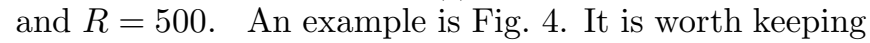
in mind at what time the boundary can have a physical influence on the observers. For $n=0$, an observer near the centre leaves the domain of dependence of the initial data at $t \simeq R_{\max }$, while any continuum reflection of the initial outgoing pulse reaches the observer

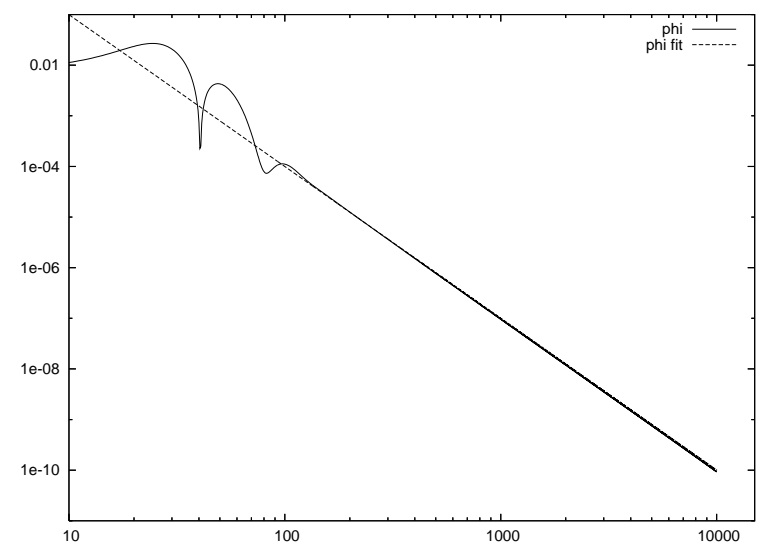

FIG. 4: Log-log plot of $\phi$ versus $t$ at $R=10$ on Schwarzschild, using $n=1$ slices, with an outer boundary $R_{\max } \sim 10^{6}$. The straight line is $t^{-3}$ with the amplitude but not the power adjusted to the data. This is the expected power-law tail. The corresponding entry in Table 1 is in bold face.

at $t \simeq 2 R_{\max }$. With $n>0$, one can roughly think of the slices as null, and of outgoing waves as going out almost instantaneously, so that both events happen close together at $t \simeq 2 R_{\max }$. We find that for $n=0$, the tail is lost at exactly the time when the continuum reflection of the outgoing wave reaches the observer (at $R=10$ and 500 respectively). For $n>0$, the tail can be lost both earlier (presumably because numerical boundary error travels faster than light) or later (presumably because at very large boundary radius the continuum reflection is small and the numerical reflection is also small).

Our best results are obtained for $n=1$ with MDBCs imposed at $R_{\max } \simeq 10^{6}$, which requires only 722 grid points for a central resolution of $\Delta R=0.1$. From the Minkowski results we see that this choice would also be among the best ones for minimising the error in the outgoing wave.

\section{CONCLUSIONS}

The essential motivation for using either the conformal field equations or null coordinates is their ability to simulate an asymptotically flat spacetime out to $\mathscr{I}^{+}$on a fi- 


\begin{tabular}{|l|c|c|cc|cc|cc|}
\hline Slice & $R_{\text {max }}$ & Grid points & \multicolumn{2}{|c|}{$t_{l}$ with MDBCs } & $t_{l}$ with outflow BCs & $t_{l}$ with buffer BCs \\
& & & $R=10$ & $R=500$ & $R=10$ & $R=500$ & $R=10$ & $R=500$ \\
\hline$n=0$ & 1000 & 10086 & 2020 & 1530 & & & $>2100$ & $>2100$ \\
\hline$n=\frac{1}{2}$ & 1000 & 884 & $\sim 1700$ & $\sim 900$ & $\sim 1800$ & $\sim 900$ & $>2100$ & $>2100$ \\
& $10^{6}$ & 28271 & $>10^{4}$ & $>10^{4}$ & $>2800$ & $>2800$ & & \\
\hline$n=1$ & 1000 & 324 & $\sim 900$ & $\sim 500$ & $\sim 600$ & $\sim 200$ & $\sim 900$ & $\sim 500$ \\
& $10^{6}$ & 722 & $>\mathbf{1 0}^{4}$ & $>10^{4}$ & $>2400$ & $>2400$ & & \\
\hline$n=\frac{3}{2}$ & 1000 & 194 & $\sim 200$ & $*$ & $\sim 200$ & $*$ & $\sim 200$ & $*$ \\
& $\infty$ & 210 & $\sim 300$ & $\sim 150$ & & & & $*$ \\
\hline$n=2$ & 1000 & 152 & $*$ & $*$ & $\sim 100$ & $*$ & $*$ & $*$ \\
& $\infty$ & 153 & $\sim 300$ & $\sim 200$ & & & & \\
\hline
\end{tabular}

TABLE II: Time $t_{l}$ when power-law tails are lost for different slicings, different BCs, two outer radii, and two observer locations. Note that $T=t+$ const for these observers at fixed $R$, where the constant depends on $R$ and the slicing. A $*$ means that power-law tails were not seen. A blank means that this combination of slice and $\mathrm{BC}$ is not sensible. $R_{\text {max }}$ is the outer radius of the physical region (defined arbitrarily as $c_{+}>1 / 2$ ), not of the buffer. With buffer BCs the number of grid points increases by about $20 \%$ from the value stated in the third column.

nite domain. However, this can be done accurately with a finite number of grid points only in situations where ingoing radiation, including backscatter, can be progressively neglected at large radius. Conversely, in any physical situation where these approaches work, one should be able to simulate the spacetime almost up to $\mathscr{I}^{+}$by using asymptotically null slices without regularising the field equations at infinity [13, 14].

From the requirement that the outgoing coordinate speed of light should neither go to zero (the code becomes inefficient and too dissipative) nor to infinity (explicit schemes become unstable, and implicit schemes are likely to become inaccurate), we have established a quantitative relationship between the rate at which the slices become null at large radius and the rate at which the radial coordinate is compactified.

This rate can be characterised by a parameter $n$ in the range $0 \leq n \leq 2$, where on the one hand the physical radius (area radius) $R$ and coordinate radius $r$ are asymptotically related by $r \sim R^{1-n}$, and on the other hand the Lorentz factor of the slices asymptotically diverges as $\Gamma \sim R^{n / 2}$ and the extrinsic curvature of the slices scales as $K \sim R^{n / 2-1}$.

The extreme value $n=2$ corresponds to the hyperboloidal slices which have traditionally been used both in drawing the standard conformal diagram of Minkowski or Schwarzschild spacetime, and in numerical evolutions using the conformal field equations. For $0<n \leq 1$ the range of the coordinate $r$ is infinite (we prefer to speak of stretching rather than of compactification) and surprisingly the slices terminate at $i^{0}$. For $1<n \leq 2$ the range of $r$ is finite and the slices intersect $\mathscr{I}^{+}$as expected.

Our numerical simulations of the spherical wave equation on Minkowski and Schwarzschild spacetimes have confirmed our expectations. For a given (very large) radius of the outer boundary and a given (realistic) accuracy requirement as the outgoing wave reaches that boundary, simulations in our $n>0$ coordinates require dramatically fewer grid points. To compare two extreme examples, evolving an outgoing wave with wavelength $\Delta R \sim 1$ out to $R=1000$ with a relative error of $20 \%$ requires 10000 gridpoints (and 25000 timesteps) in standard coordinates using 4th-order finite differencing, while only 165 grid points (and 390 time steps) with our $n=2$ coordinates give a relative error of only $4 \%$.

Although $n>0$ coordinates do not represent ingoing waves accurately, power-law tails of waves on Schwarzschild are correctly represented, until numerical error from the boundary becomes bigger than the tail signal. Lower values of $n$ represent the tails correctly for longer time because numerical error from the boundary propagates in less rapidly. This suggests that in applications to numerical relativity all values of $n$ should be considered. From our numerical experiments, the preferred value of $n$ as a compromise between minimising grid size, representing outgoing waves, and representing power-law tails is $n=1$. It also appears that an $n=2$ slicing would have to be adapted to the angular momentum of the central source, whereas $n<2$ slicings are only affected by the mass.

In $3 \mathrm{D}$, our method requires approximately constant angular resolution as $R \rightarrow \infty$. A promising technique for achieving this while avoiding the axis singularity of spherical polar coordinates is the use of multiple coordinate patches [19, 20, 21, 22].

In summary, our proposed approach for pushing the BCs to very large radius requires only a modification of the initial data and the gauge conditions but uses standard $3+1$ field equations. It is simpler than the conformal field equation method and Cauchy-characteristic matching methods. Although it cannot reach $\mathscr{I}^{+}$, it can represent outgoing waves out to arbitrarily large radius on a finite grid. Our results also suggest certain improvements to existing numerical relativity codes: 
a) A lower value of $n$, perhaps $n=1$, may be better than $n=2$ for representing physically interesting scenarios accurately. The conformal and null methods require $n=2$ because they require a regular conformal spacetime metric, and this requires the conformal factor $\Omega \sim R^{-2}$ as $\mathscr{I}^{+}$is approached. Keeping this asymptotic behaviour, one may be able to improve their accuracy at large $R$ by using a smaller value of $n$ out to some very large value of $R$ and then switching to $n=2$ to reach $\mathscr{I}^{+}$.

b) In 3D implementations of compactification at $i^{0}$ [5, 12], Cartesian spatial coordinates are compactified as $X^{i}=\tan x^{i}$, which corresponds to $n=2$ compactification in our notation. Our results suggest a modification of this approach in which the slices are made asymptotically null up to large finite radius, perhaps best with $n=1$. The resulting code would represent outgoing radiation correctly out to this large finite radius. Outside this region the slice could still reach $i^{0}$, for example by switching to $n=2$ (the traditional value).

c) Fixed mesh refinement $[\underline{6}, \mathbf{7}$, , 8], in 3D typically using Cartesian grids, is also widely used to push the outer boundary further out. If this is done with nested boxes with equal (or comparable) numbers of grid points in each box, one automatically has approximately constant angular resolution, and radial resolution $\Delta R \sim R$, which corresponds to $n=1$ stretching in our notation. Our results suggest combining the nested boxes approach with $n=1$ slicing (and a global time step), so that outgoing waves are resolved all the way out to the outer boundary, which can then be put at arbitrarily large radius.

\section{APPENDIX A: CLOSED FORM COORDINATE TRANSFORMATIONS}

We give closed-form functions $R(r)$ and $F(R)$ which have the required asymptotic behaviour (31) and (38). For the radial stretch we choose

$$
R(r)=r\left(1+\frac{r^{2}}{l^{2}}\right)^{\frac{n}{2(1-n)}}, \quad l=\left(\frac{1}{1-n}\right)^{1 / n} L
$$

for the generic case $0<n<1$, and

$$
R(r)=L \sinh \frac{r}{L}
$$

for $n=1$. The range of $r$ is $0 \leq r<\infty$. For the compactification we choose

$$
R(r)=r\left(1-\frac{r^{2}}{l^{2}}\right)^{\frac{1}{1-n}}, \quad l=\left(\frac{2}{n-1}\right)^{1 / n} L
$$

for $1<n \leq 2$. The range of $r$ is now $0 \leq r<l$.

The expression for $F(R)$ can be assembled from the functions

$$
G_{n}(R, L)=\frac{L^{n}}{1-n}\left(R^{2}+L^{2}\right)^{\frac{1-n}{2}}-\frac{L}{1-n}
$$

for $0 \leq n \leq 2$ with $n \neq 1$ and

$$
G_{1}(R, L)=\frac{L}{2} \ln \left(1+\frac{R^{2}}{L^{2}}\right)
$$

for the special value $n=1$. They obey

$$
G_{n}^{\prime}(R, L)=\left(\frac{R}{L}\right)^{-n}+O\left(R^{-n-2}\right) .
$$

for all $n$ including $n=1$. In particular,

$$
G_{0}^{\prime}(R, L)=1-\frac{L^{2}}{2 R^{2}}+O\left(R^{-4}\right),
$$

and the $R^{-2}$ term in $G_{0}^{\prime}$ has to be taken into account in the $n=2$ slicing. It is straightforward to construct $F(R)$ from these blocks. We only give an example here. For $0<n<2$ with $m=q=0$, we can use

$$
F(R)=G_{0}\left(R, L_{0}\right)-\left(\frac{L_{n}}{L}\right)^{n} G_{n}\left(R, L_{n}\right) .
$$

Note that $L_{0}$ and $L_{n}$ can differ from each other and from $L$ (the constant introduced in $R(r)$ ) as long as the corresponding amplitude is adjusted. For $n \geq 1$ with $m>0$ and again for $n=2$, additional terms have to be added. Similarly, to achieve a switchover from $n$ to $n_{\infty}<n$ at a given large $R$ we only need to add $G_{n_{\infty}}$ with suitable amplitude and scale.

\section{APPENDIX B: WAVE EQUATION AND BOUNDARY CONDITIONS}

We write the massless wave equation

$$
\nabla_{a} \nabla^{a} \phi=0
$$

in a $3+1$ form similar to the ADM form of the Einstein equations by defining

$$
\Pi \equiv-\mathcal{L}_{n} \phi=-n^{a} \nabla_{n} \phi,
$$

where $n_{a}$ is the future-pointing unit normal on the slices of constant $t$. With $t^{a} \equiv(\partial / \partial t)^{a} \equiv \alpha n^{a}+\beta^{a}$ this gives

$$
\begin{aligned}
& \mathcal{L}_{t} \phi=\mathcal{L}_{\beta} \phi-\alpha \Pi, \\
& \mathcal{L}_{t} \Pi=\mathcal{L}_{\beta} \Pi-\alpha D_{a} D^{a} \phi-\alpha a^{b} D_{b} \phi+\alpha K \Pi .
\end{aligned}
$$

Here $D_{a}$ is the covariant derivative associated with the three-metric $\gamma_{a b}$ on each slice, and $a^{b}=D^{b} \ln \alpha$ is the acceleration of the $n^{a}$ observers.

At the outer boundary the MDBC corresponding to no incoming radiation [23] would be,

$$
\Pi-m^{a} D_{a} \phi=0,
$$

where $m^{a}$ is the outward-pointing unit vector normal to the boundary within each constant $t$ slice. In Minkowski 
this is equivalent to $\phi=f(R-T)$. By contrast an outgoing spherical wave in Minkowski is $\phi=f(R-T) / R$. We can achieve this boundary condition by adding a lowerorder term to the MDBC, namely

$$
\Pi-m^{a} D_{a} \phi+Q \phi=0, \quad Q \equiv \frac{\alpha-\beta^{r} \sqrt{\gamma_{r r}}}{\sqrt{\gamma_{\theta \theta}}} .
$$

Note that a MDBC is defined to lead to a non-increasing energy in the frozen coefficient, principal part only approximation, so that this modified $\mathrm{BC}$ is still a MDBC.

Following [17], we discretise this to 2nd-order accuracy as

$$
\begin{aligned}
\Pi_{N}-\sqrt{g^{r r}} D_{0} \phi_{N}+Q_{N} \phi_{N} & =0 \\
h^{3} D_{-}^{3} \Pi_{N+1} & =0
\end{aligned}
$$

where $N$ is the grid point at $r=r_{\max }$ and $N+1$ is a ghost point. For 4-th order accuracy we use

$$
\begin{aligned}
\Pi_{N}-\sqrt{g^{r r}} D^{(1)} \phi_{N}+Q_{N} \phi_{N} & =0, \\
h^{5} D_{+}^{5} \phi_{N+2} & =0,
\end{aligned}
$$

$$
\begin{aligned}
& h^{4} D_{+}^{4} \Pi_{N+1}=0, \\
& h^{4} D_{+}^{4} \Pi_{N+2}=0,
\end{aligned}
$$

where $D^{(1)}$ is defined by

$$
D^{(1)} \equiv D_{0}\left(1-\frac{h^{2}}{6} D_{+} D_{-}\right)
$$

At the black hole-excision boundary, or where the outer boundary is null or spacelike, no BCs are required for the continuum equations. As numerical BCs we then use extrapolation for all ghost points including $\phi_{N+1}$, that is

$$
h^{5} D_{+}^{5} \phi_{N+1}=0
$$

in the 4 -th order accurate case, and $D_{+}^{3}$ in the 2 nd-order accurate case.

Our artificial dissipation operators are $-\sigma h^{3}\left(D_{+} D_{-}\right)^{2}$ at 2nd-order accuracy and $\sigma h^{5}\left(D_{+} D_{-}\right)^{3}$ at 4 th order. We use extrapolation to populate the additional ghost points required at boundaries.
[1] H. Friedrich and G. Nagy, Comm. Math. Phys. 201, 619 (1999).

[2] G. Calabrese, L. Lehner and M. Tiglio, Phys. Rev. D 65, 104031 (2002).

[3] L. E. Kidder et al., Phys. Rev. D 71 064020, (2005).

[4] O. Sarbach and M. Tiglio, Journal of Hyperbolic Differential Equations 2, 839 (2005).

[5] F. Pretorius, Class. Quant. Grav. 22, 425 (2005).

[6] U. Sperhake et al., Phys. Rev. D 71, 124042 (2005).

[7] L. Baiotti et al., Phys. Rev. Lett. 94, 131101 (2005).

[8] M. Miller, Phys. Rev. D 71, 104016 (2005).

[9] J. Frauendiener, Living Reviews in Relativity 2004-1.

[10] J. Winicour, Living Reviews in Relativity 2005-10.

[11] E. P. Honda and M. W. Choptuik, Phys. Rev. D 65, 084037 (2002).

[12] D. Garfinkle and G. C. Duncan, Phys. Rev. D 63, 044011 (2001).

[13] A. Kansagra, Coordinate compactifications and hyperboloidal slices in numerical relativity, LIGO/Caltech undergraduate project, unpublished (2003). For an online abstract, see http://www.ligo.caltech.edu/LIGO_web/ students/ugprojects03.html.

[14] C. Misner, M. Scheel, Wave propagation with hyperboloidal slicings, talk given 12 June 2003 at KITP, unpublished. For an online version see http://online.kitp.ucsb.edu/online/gravity03/misner/ C. Misner, Hyperboloidal slices and artificial cosmology for numerical relativity, e-print gr-qc/0409073 J. R. van Meter, D. R. Fiske, C. W. Misner, Excising das All: Evolving Maxwell waves beyond Scri, e-print gr-qc/0603034

[15] S. W. Hawking and G. F. R. Ellis, The large scale structure of spacetime, Cambridge University Press, 1975.

[16] D. Garfinkle and C. Gundlach, Class. Quant. Grav. 16, 4111 (1999).

[17] G. Calabrese and C. Gundlach, Discrete boundary treatment for the shifted wave equation, e-print gr-qc/0509119 to be published in Class. Quant. Grav.

[18] R. Price, Phys. Rev. D 5, 2419 (1972).

[19] G. Calabrese and D. Neilsen, Phys. Rev. D 69044020 (2004)

[20] G. Calabrese and D. Neilsen, Phys. Rev. D 71124027 (2005).

[21] J. Thornburg, Class. Quant. Grav. 21, 3665 (2004).

[22] L. Lehner, O. Reula and M. Tiglio, Class. Quant. Grav. 22, 5283 (2005).

[23] B. Gustafsson, H. Kreiss, and J. Oliger, Time dependent problems and difference methods, John Wiley \& Sons, New York, 1995. 